\title{
ACCESSIBLE COMPUTER INTERACTION FOR PEOPLE WITH DISABILITIES The case of quadriplegics
}

\author{
Paula Kotzé, Mariki Eloff, Ayodele Adesina-Ojo \\ School of Computing, University of South Africa, Pretoria, South Africa \\ Jan Eloff \\ Department of Computer Science, University of Pretoria, Pretoria, South Africa
}

Keywords: Accessibility to disabled users, Accessibility options and technology, Quadriplegics, User needs

\begin{abstract}
Universal design is the design of products and environments so that anyone can use them without adaptation or specialised design. Life must be simplified by making products, communications and the built environment more usable for as many people as possible at little or no extra cost. To understand the challenges that a disabled person has to face when using the computer, we have to know what capabilities such a person has. Only then will it be possible to apply universal design to computer interfaces. The purpose of this paper is to highlight the challenges that many people face in their everyday lives and determine to what extent disabled people, especially people with limited or no use of their hands and arms, interact independently with computer equipment. The paper specifically looks at quadriplegics, their capabilities, a survey of how they use computer equipment, as well as special devices available to assist them in this interaction.
\end{abstract}

\section{INTRODUCTION}

The World Health Organisation (WHO) defines a person with a disability (or activity limitation) as a person of any age who is unable to perform, independently and without aid, basic human activities or tasks, due to a health condition or physical/cognitive/psychological impairment of a permanent or temporary nature (Sandhu, 2003). According to the World Bank estimate, $10 \%$ of the world's population (approximately 600 million people) suffer from some form of disability (Metts, 2000). The United Nations (UN), on the other hand, prefers to identify the percentage of disabilities on a country-to-country basis, as each country uses different screening techniques during surveys or censuses conducted for people with disabilities (UN Statistics Division, 2003). From the UN disability report, it can be seen that the First World countries appear to have a much higher percentage of disabled persons than do the Third World countries. For example, Norway (1996) and New Zealand (1996) record the ratios of $33 \%$ and $20 \%$ respectively. On the other hand, Nigeria (1991) and South Africa (1980) both documented $0.5 \%$ of their population as having certain limitations. This can be attributed to the fact that many of the Third World countries have only rough estimates based on infrequent censuses.

'In a fair society, all individuals would have equal opportunity to participate in, or benefit from, the use of computer resources regardless of race, sex, religion, age, disability, national origin or other such similar factors' (ACM Code of Ethics: http://www.acm.org/constitution/code.html). Using computers is becoming increasingly important in everyday life, not only in the work environment, but also in our homes. Whether for work or leisure, our interaction with computers should be as comfortable and productive as possible. As a minimum requirement, users must be able to view the display, reach the input devices, etc. This is especially true for people with disabilities (Shneiderman, 1993). A number of factors may intervene to restrict or prevent users with disabilities from achieving this. 
User interfaces often reflect the assumptions that their designers make about the physiological characteristics of their users. Interaction objects are designed so that an 'average' user can easily manipulate (select, copy, move, etc.) them by means of a mouse, tracker-ball, touch-pad, cursor keys, etc. Unfortunately the concept of an 'average user' is a myth (Kotze, 2000). Some users, for example, have the physiological ability and capacity to make finegrained selections, but others, such as quadriplegics, do not.

Do we always realise how frustrating it might be for a person not to be able to switch on a computer, let alone insert or remove a diskette? People with physical limitations may not be able to use these standard input devices effectively and may benefit from using special devices or software, for example, speech recognition software (Loy \& Batiste, 2001). Using the keyboard may require a special device, allowing the user to press only one key at a time. To date, disabled people have a tendency to adapt to technology, and they do not always require or even demand that technology must be adapted to their needs (Nordic Guidelines for Computer Accessibility, 1998). New legislation, based in many cases on rights entrenched in constitutions and bills of human rights, is changing this tendency.

Universal design is the design of products and environments so that anyone can use them without adaptation or specialised design (Polk, 2000). The increased pressure for universal access and usability is a happy by-product of the growth of the Internet. Services using electronic communication and the Internet, for example e-commerce, e-learning, healthcare informatics, international and national travel, financial systems, mobile communication, etc., are expanding rapidly and users are becoming dependent on them. Critics of information technology abound, but they often focus on the digital divide of an information-poor minority, with far less emphasis on the physically and cognitively challenged (Shneiderman, 2002). To understand the challenges that a disabled person has to face when using the computer, we have to know what capabilities such a person has. Only then will it be possible to apply universal design to computer interfaces.

The purpose of this paper is to investigate and report on the requirements, status and available technology to assist quadriplegics in their interaction with computing devices. Section 2 gives a brief overview of capability levels of quadriplegics. Section 3 addresses quadriplegic human-computer interaction and assistive technology aimed at quadriplegics. It gives the results of a survey as to how people with limited or no hand or finger movement interact with computers, followed by suggestions on possible improvements. Section 4 constitutes a conclusion to these considerations.

\section{CAPABILITIES OF QUADRIPLEGICS}

Human limitations related to computer interaction can be grouped into five categories (Microsoft Accessibility, 2003; Vanderheiden, 1994):

1. Resource limitations refer to the inability of people to have access to education and infrastructure that would better their quality of life.

2. Learning limitations describe the lack of processing abilities amongst certain people, which interferes with their learning process. Such persons typically suffer from dyslexia and attention deficit disorders, amongst other limitations, and may require individualised course presentations.

3. Hearing limitations mean that people may experience varying degrees of auditory loss, ranging from slight hearing loss to deafness.

4. Visual limitations include low vision, colour blindness and blindness. People with these impairments have to rely heavily on other senses such as touch and sound.

5. Mobility limitations affect people stricken by certain illnesses or affected by accidents that deny them the full use of their limbs, who therefore have difficulty in holding and reaching for objects or moving around.

This paper will focus only on factors and issues that are directly related to mobility impairments in general, and in particular, those factors that are more likely to affect the use of hands and upper body parts, with special reference to quadriplegia. Quadriplegia, or paralysis from the neck down, can have many causes. After an introduction to the anatomy of the spinal cord in Section 2.1, some of the possible causes of paralysis from the neck down are briefly mentioned in Section 2.2.

\subsection{Anatomy}

The central nervous system (CNS) consists of the brain, spinal cord and nerves. It is in charge of most functions of the body and mind, which include voluntary movements like walking, as well as involuntary movements like blinking. The brain interprets messages from the sensory organs such as the eyes, nose, skin and tongue, muscles as well as internal organs. The spinal cord (SC) acts as the information highway between the body and the brain, and it is divided into 5 main sections, made up of 33 
vertebrae that are separated by spongy disks. Each main section consists of subsections that are mapped to different parts of the body (with some degree of overlapping occurring). The first eight segments of the spinal column at the top of the spine are called 'cervical vertebrae' and are named C1 to C8. The next 12 vertebrae of the upper back are the thoracic vertebrae, named T1 to T12. T1 lies just below the eighth cervical vertebra (C8) and T12 lies just above the first lumbar vertebra (L1). There are five lumbar vertebrae, L1 to L5, followed by three sacral vertebrae and the coccygeal (CRPF, 2003; Mosby's Medical Encyclopaedia, 1997).

Figure 1 depicts this mapping. Although a similar organization exists for the SC mapping to internal organs (for hormone release, etc.), it is not discussed here. Any injury to the cervical vertebrae will affect the shoulders, arms and/or hands. The lower the injury, the more functionality the person will have. A C6/C7 injury may leave a person with full use of his shoulders and arms, but no movement of his fingers. A person with all four limbs affected is called a quadriplegic (or a tetraplegic). A person with an injury to the thoracic vertebrae will be affected from the chest down and is termed a paraplegic. Paraplegics usually have full use of their arms and hands, and can use the computer in the same way as any other able-bodied person. Diplegia is the bilateral paralysis of similar parts of the body. The research reported upon in this paper focuses on how people with less than full use of their upper limbs interact with the computer and what needs to be done to improve their interaction.

\subsection{Diseases and Injury}

\subsubsection{Spinal Cord Injuries}

Spinal cord injuries (SCI) are the result of traumatic or non-traumatic causes, leading to disruption in the exchange of information between the brain and the body, resulting in paralysis (CRPF, 2003). The trauma can result in severed nerves, pressure on and bruising of the spinal cord. Paralysis causes loss of sensation and control over the voluntary movement and muscles of the body. With SCI, damage to the spinal cord will only affect areas below the point of injury. Long-term disability depends on the severity of the injury, in which nerve fibres are damaged, and the part of the SC that is injured (National Institute of Neurological Disorders and Strokes, NINDS, 2003). Traumatic injury occurs most often in young adult men, and non-traumatic injury is more common in individuals over 50 years of age. Spinal cord tumours, spondylosis and vertebral disk degeneration are common non-traumatic causes of spinal cord injury (Mosby's Medical Encyclopedia, 1997). Annually, about 55 million people suffer from SCI, with about 35 million surviving the acute injury. Worldwide there are an estimated 500 million to 900 million survivors of SCI. In South Africa, the leading causes of SCI are vehicle and sporting accidents, as well as gunshot-related (violent) injuries (QASA, 2003). There are an estimated 64000 people in South Africa who are in wheelchairs (Marais, 2001). More than half of the individuals are quadriplegic, and the remainder paraplegic.

\subsubsection{Multiple Sclerosis}

The nerves in the CNS permit smooth and coordinated movement with little conscious effort. A fatty tissue, called myelin, surrounds and protects the nerve fibres of the CNS. Myelin is also responsible for conduction of electric pulses produced by the nerve fibres. Multiple Sclerosis (MS) occurs when demyelination (loss of myelin) occurs, resulting in areas of the CNS hardening and the formation of scars or lesions, hence the name 'multiple sclerosis' meaning 'many scars' (MSIF, 2003). The result is that impulse conduction of the nerves is slowed or halted. The symptoms of MS include, inter alia, chronic fatigue, loss of balance and/or muscular coordination known as 'ataxia', numbness of body parts such as hands or feet hampering the ability to hold objects or walk properly. Causes of MS are mostly unknown, although a person's gene pool may contribute (but not directly) to the predisposition. A person's gender may also play a part, with 2 to 3 more women than men likely to be afflicted by the disease. The disease also has a bias for young adults in the 20-40 year age group and rarely affects children less than 12 years old or elderly people over 55. Finally, the racial group of northern European people is more likely to suffer from the disease, although Asian, African or Hispanic race groups are not immune to it.

\subsubsection{Hemiplegia, Hemiparesis and Cerebral Palsy}

Hemiplegia is the total paralysis of the same side of the body (CHSA, 2003). It differs from hemiparesis, which is weakness in one side of the body. The most common reason for this is a disruption of blood flow to the brain, resulting in part of the brain dying. When this occurs, the part of the body that is controlled by the damaged part of the brain will be weakened. Paralysis occurs in the side of the body opposite to the affected part of the brain, that is, if the left side of the brain is affected by insufficient 
blood flow, the right side of the body will be paralysed, and vice versa. Common causes or contributing factors of hemiplegia are infections such as meningitis, brain abscesses, diabetes, high levels of cholesterol, penetrating and nonpenetrating head trauma, and congenital injuries. Symptoms of hemiplegia other than paralysis include loss of sensation in body parts, imbalance weakness, tremors and unsteadiness. When paralysis is diagnosed in children, the condition is called 'cerebral palsy'. Juvenile paralysis may produce hemiplegia, paraplegia, quadriplegia or diplegia (CHSA, 2003).

\subsubsection{Amyotrophic Lateral Sclerosis}

Amyotrophic Lateral Sclerosis (ALS), or Lou Gehrig's disease, is a disorder of the parts of the CNS that control the voluntary muscles in charge of voluntary movements (ALSA, 2003). The disease targets the upper and lower motor neurons. Upper motor neurons send chemical messages to the lower motor neurons, which in turn send the messages to muscles in the limbs, trunk, head and respiratory system. Malfunctioning neurons cause muscles to become weakened, severely affecting mobility. ALS is a progressive disease with continued loss of function, often resulting in death in as little as 5 years after the onset of symptoms. Very rarely do people with this disease survive for longer than 10 years after onset. The symptoms usually begin in one arm or leg, spreading to the other until the entire body is totally ravaged by the disease, resulting in complete paralysis. This disease affects adults more than teenagers, even though its occurrence in young people cannot be excluded.

\subsubsection{Muscular Dystrophy}

Muscular Dystrophy (MD) is a name given to a group of diseases that are hereditary and often manifest as non-rapid progressive wasting of muscles that control body movement (MDAC, 2003). This causes a characteristic, selective pattern of weaknesses and thus targets different parts of the body with different people. Muscular dystrophy is not contagious. The clinical onset may begin at any time in childhood or adulthood. For example, Duchenne MD is often detected in children between the ages of 2-6 years and affects only boys. Distal $\mathrm{MD}$, on the other hand, develops in adults in the 4060 year age group. In cases where the mother is the carrier, her sons are more likely to be infected and her daughters will only be carriers of the disease. Although this disease has no cure, therapy can help to maintain a certain level of independence with a normal life span.

\subsubsection{Repetitive Stress Injuries}

The term 'repetitive stress injury' (RSI), or 'repetitive strain', refers to a group of conditions caused by placing too much stress on a joint. Repetitive stress injury happens when the same action is performed repeatedly (RSIA, 2003). When an action that is stressful to a joint is repeated frequently, the area does not have time to recover and it becomes irritated. This can cause the area to become painful and swollen. One of the most common types of repetitive stress injury is carpal tunnel syndrome. The median nerve is the most sensitive nerve that travels down the arm to the hand to the thumb, index, middle and half of the 'ring' fingers. It passes through a narrow path (a tunnel) at the wrist. The ailment 'carpal tunnel syndrome' occurs when bones and ligaments in the wrist joint causing tingling sensations or numbness, compress the median. If left untreated, it can cause complete loss of feeling in most of the fingers as nerves become damaged. Carpal tunnel syndrome can affect anyone, but mostly occurs in women aged 40-45 years and among people involved in heavy labour.

\subsubsection{Parkinson's Disease}

Parkinson's disease is a progressive disorder of the CNS (The Parkinson's Web, 2003). It causes muscle stiffness and rigidity, tremors and slowness of movement. The main cause of Parkinson's disease is unknown, although there are unverified claims that it is linked to inherited genes and environmental triggers. The disease begins when there is a shortage of a brain-signalling chemical called dopamine. Symptoms are initially subtle but worsen within 3-5 years as the disease progresses, until eventually no medication can control any advancement. The disorder is not contagious and very seldom affects people younger than 30 years old, but likelihood drastically increases with age, especially after fifty.

\subsubsection{Arthritis}

Arthritis is the inflammation of joints causing swelling, stiffness and difficulty in the movement of fingers, wrists, knees and hips, amongst other body parts (AA, 2003). There are two main categories, namely osteoarthritis (OA) and rheumatoid arthritis (RA). Causes of both forms of arthritis are largely unknown, although OA is attributed to the release of too many enzymes that cause the joint cartilage to break down. Eventually, the cartilage wears out completely, the joints start to rub together, and the bone loses its shape and juts out, forming lumps. $\mathrm{RA}$, on the other hand, may be triggered by 
inflammation of the joint lining instigated when white blood cells migrate to the capsule that contains lubrication fluid for joints. OA affects more women than men over the age of 45 and especially after menopause, while RA affects more men than women at an age younger than 45 . Juvenile cases of arthritis have also been identified, with children as young as a few months being stricken by the ailment (CHSA, 2003).

\section{QUADRIPLEGIC HUMAN- COMPUTER INTERACTION}

As mentioned in the introduction, in this paper we will specifically focus on quadriplegics. Quadriplegics (quads) are in wheelchairs. The first computer interaction obstacle they need to overcome is the physical position of the CPU, screen and keyboard. Does the wheelchair fit comfortably under the desk or table? Can the user reach and use the keyboard with ease? Is it possible to position the CPU box in such a way that the user can switch the computer on/off? If it is too high or to low, it might be difficult to reach. The same applies to using the diskette drive and CD ROM.

A quadriplegic may not have full functional use of his/her hands and may use the keyboard with a splint or special strap on one hand that holds a stick to press the keys. The one end of the stick is generally covered with a piece of sponge or rubber to improve typing, otherwise the stick might slip off the desired key. As can be expected, the typing speed may be quite slow. Some users may use a stick in their mouths on the keyboard. Holding the 'shift' or 'control' key down and then pressing another key with a stick in one hand is just short of impossible. Using the mouse may also be an uphill battle for some users - to be able to move the mouse to the desired position and then 'click' using both hands with limited finger function might not be very easy, let alone comfortable. Different pointing devices do give the user a choice. Notebooks come with built-in pointing devices - a touch-and-drag type of mouse, track ball or even a little peg in the keyboard. An 'external' mouse can be connected to a laptop, if desired.

\subsection{Survey on how quadriplegics interact with computers}

Before any suggestions on improving computer interaction for disabled people can be made, we need to determine exactly how disabled people currently interact with computers. A survey was conducted amongst quads with limited functionality in their hands, wrists or arms. The aim of the survey was to find out what they can do when using the computer and how they do it. A total of 20 quads were interviewed, with disability causes ranging from spinal cord injuries, polio, cerebral palsy to muscle dystrophy. The following summarises the major findings:

- $82 \%$ suffered from spinal cord injuries, $6 \%$ from muscular dystrophy, $6 \%$ from cerebral palsy and $6 \%$ were disabled due to childhood polio.

- $25 \%$ could not switch the computer on or off, while $31 \%$ could not use the diskette drive effectively, and $38 \%$ of the users could not use the CD-ROM. Seventeen per cent into all three categories.

- The keyboard was used by operating a stick in a splint by $41 \%$ of the users (all with spinal cord injuries), $44 \%$ by one hand or fingers from one hand and 19\% with both hands, or fingers from both hands.

- $88 \%$ of the users used a mouse. Twenty-six per cent of these used both hands, while the remainder used the mouse with only one hand. Sixty-six per cent of these used an ordinary mouse, while the others used a trackball type mouse or a touchpad.

- Only 56\% knew about sticky keys, while only $25 \%$ used them.

- $63 \%$ of the respondents used a computer before their disability.

- $40 \%$ of the respondents were male and $60 \%$ female, ranging in age from late teens to midfifties, with all having completed high school and $81 \%$ having a tertiary degree qualification.

Some of the specific problems that were mentioned include the following:

- It is too difficult to change the volume control on the speakers - even if they are within reach. The buttons are too small and too stiff to turn with limited or no finger movement.

- It would help if the keys were more sensitive to touch and easy to press - they are sometimes too stiff. Even a keyboard with the keys wider apart may make a difference.

- An easier way to switch the computer on or off what about using the keyboard?

- Predictive text would also help, provided it functioned correctly.

- Voice recognition is not the answer for all - two of the seven people who used voice recognition software were highly frustrated with it and either stopped using computers or reverted back to the keyboard and mouse.

- Few users who used the keyboard knew about sticky keys or the other accessibility options in Windows. 
One major item of feedback was the fact that all the people interviewed adapted to the computer technology, and did not require the technology to adapt or be changed to suit their needs. The reason for this can be twofold. First is the lack of knowledge that other possibilities may exist - they use what is available without searching for other solutions or even requesting, let alone demanding, that the technology be changed to suit their needs. When the users were asked what they would like to have changed, there were few requests. Secondly, there seems to be a lack of customer support, especially regarding the voice recognition software.

\subsection{Suggestions on possible improvements}

The flexibility of computer software makes it possible for designers to provide special services for users with disabilities. Designers can benefit by planning early on to accommodate users with disabilities and work more closely with computer users with special needs in order to design what will suit them (Kotze, 2000). It is, however, pertinent that new technology options be communicated to users with special needs.

Assistive technologies or electronic curb cuts (from the term curb-cut in city sidewalks to facilitate wheelchair mobility) are technologies that are needed to enhance disabled persons' independence, self-esteem and over-all quality of life (UN, 2003). Assistive technologies developed specifically for computer technology are required to allow users to have seamless access to computer technology and, more importantly, to permit full customisation. Full customisability of computer assistive technologies is required as disabilities grouped even within the same category may differ by varying degrees, as was indicated by the results of our survey. Although assistive technologies were initially developed for people with various limitations, many of the developments, such as remote controls, have inevitably benefited everyone (TIA, 1996). This should therefore be an incentive for further research into the development of newer and more comfortable equipment. Apart from humanitarian reasons, other incentives for producing accessible products include compliance with standards and regulations laid down by organisations such as the UN and the WHO. Economic factors also buttress the argument for assistive technologies. As stated before, according to the World Bank, $10 \%$ of the world's population suffers from some form of disability, therefore increasing the potential customer base (which is big already). Assistive technology also means that there is no loss of expertise or revenues for the organisations with an aging population, and employees with temporarily or permanently acquired disabilities would have a trouble-free reintegration into the workplace after recuperation and rehabilitation (NGCA, 1998). Our survey indicated that $81 \%$ of the respondents had a degree qualification, while $63 \%$ of these had a postgraduate qualification, and were therefore potentially highly valuable to the workforce.

Standard hardware and software technologies provided by information technology vendors often provide inexpensive access features that can be used by persons with less severe cases of motor disability. Therefore, before discussing specific hardware and software developed for quadriplegia, an overview is given of access features provided by status quo technologies.

\subsubsection{Status Quo Technologies}

The Dvorak keyboard was designed to provide an alternative keyboard arrangement to the standard QWERTY keyboard. The commonly used letters (namely the vowels 'AOEUI' and most common consonants 'DHTNS') of the English language are arranged on the 'home' row. The rationale behind this arrangement stems from the fact that, with these letters being the most commonly used, a keyboard arrangement to accommodate them greatly decreases the amount of hand-motion required to type documents (Grassie, 2001). In the light of this, some existing operating systems (OS) provide the ability for one to convert from the standard QWERTY arrangement to that of the Dvorak. The software required to reconfigure a keyboard is sometimes pre-installed in the OS, or may be downloaded or purchased from the appropriate organisation. Following the conversion of the keyboard configuration, the key-caps are popped off and re-arranged to reflect the new configuration. As an alternative, some organisations such as Microsoft ${ }^{\circledR}$ (Microsoft Accessibility, 2003) provide free downloadable stickers to be placed over the existing keyboard layout, thus eliminating the need to change a keyboard's layout physically.

Application programs provide keyboard shortcuts for persons who are able to type on keyboards but lack the dexterity required to use alternative input devices such as the mouse. To facilitate menu activation and other functionalities such as saving a file, a single key or a combination of keys can be employed in accomplishing such tasks. As examples, Microsoft ${ }^{\circledR}$ application programs use CTRL + SHIFT $+<$ to decrease the font size of characters. Similarly, with Apple ${ }^{\circledR}$ computers, the keyboard 
combination COMMAND + SHIFT +1 is used to eject a diskette from its drive. Also available are mechanisms to change default shortcuts provided by software by either creating new shortcuts or deleting some default values that one considers redundant (Dallabrida, 2002).

To further decrease the discomfort sometimes associated with using the keyboard, other complementary access functions are available. 'Sticky keys' is a software latch that enables one to depress a combination of modifier keys in sequence rather than simultaneously, and have it remain active until a non-modifier key is pressed. This feature is extremely useful for mobility-impaired persons, such as quadriplegics, as keyboard shortcuts can be used more easily. Slow keys permit users to alter the length of time taken for keystrokes to be registered on the screen. This allows accidental key depression to be cancelled without any adverse effects. Toggle keys alert the user by sounding a signal when the caps lock, num lock or scroll lock key is activated. This ensures that the user is informed about accidental key depressions. Filter keys allow brief or repeated keystrokes to be ignored, or slow down the repeat rate. Finally, 'mouse keys' is a program that permits one to control cursor movements via the keyboard, thereby allowing persons who lack the deftness required for mouse manoeuvring to simulate the action. The keypad numbers on a keyboard become compass points and numbers such as ' 5 ' act as the mouse button, ' 8 ' is used to move the cursor upwards, the number ' 2 ' moves the cursor downwards, etc.

Unfortunately, very few users know about these features. The ideal would be to communicate this information to all possible users who would benefit from using the accessibility options.

\subsubsection{Assistive Hardware Technology for Quads}

Although each technology is presented individually, it is often the case that more than one of these technologies are used together in order to accomplish tasks. The hardware technology (and software technology in the next section) addressed here is specifically geared towards quads. Several other technologies aimed at other forms of mobility impairments and disabilities also exist. In addition, some of the following technologies are not confined to disabilities involving mobility alone, but are equally applicable to other disabilities such as visual impairments.

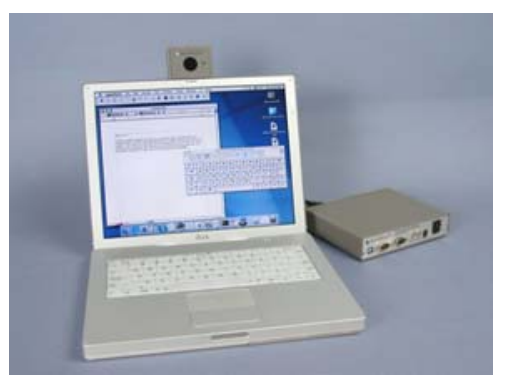

Figure 1: Head mous

(http://orin.com/access/headmouse/phm.htm)

\subsubsection{Switch/Morse Code Interface}

Switches can be controlled by almost any body part and activated with a press, kick, swipe, head movement, eye blink, and sip and puff devices. It can be mounted on a wheelchair, for example, allowing close proximity to the user. The Morse code interface is perhaps the most popular switch interface in that users can quickly adapt to using Morse code and can achieve high entry speeds. See Figure 2.

\subsubsection{Head Mouse}

A head mouse is a head-mounted wireless optical tracking system. Users' head movements are translated into proportional movements of the mouse pointer. On-screen items (e.g. icons) are selected when the cursor is left stationary on the item for a programmable length of time. See Figure 1.

\subsubsection{Eye-tracking Systems}

An eye-tracking system is an alternative input device for hands-free text entry and pointing operations. The main advantage thereof is that it requires no head-mounted technology. A camera is mounted on the monitor and focused on the eye, while integrated software gauges where the user is looking. Clicks are made either with slow blinks or with hard stares. The disadvantage of this device is that it requires the user to hold his /her head steady. See Figure 4.

\subsubsection{Sip and puff systems}

Sip and puff systems are activated by users' breath. Puffs generate a click or selection, thereby 'replacing' the need for mouse buttons. See Figure 5.

\subsubsection{Assistive Software Technology for Quads}

\subsubsection{Keyboard Filters}

Keyboard filters, such as the KeyRep Program (Nanopac Inc., 2003), are hands-on devices used for word prediction, abbreviation-expansion, 
customizable dictionaries, and decreasing the number of required keystrokes.

\subsubsection{On-screen keyboard}

On-screen keyboards, such as OnScreen v. 1.75, are devices where an image of a standard or modified keyboard is provided on the screen. Keyboard keys can be activated by practically any of the pointing devices. For people who can move the mouse cursor but cannot click, the software can be programmed to allow key selection after a certain length of time. See Figure 6.

\subsubsection{Voice/Speech Recognition Technology}

Voice/Speech Recognition Technology, for example Dragon NaturallySpeaking ${ }^{\circledR}$, consists of hands-free devices that users can use to produce text or activate functions just by speaking (Nanopac Inc., 2003). They relieve the users of the physical exertion required by direct hands-on input.

\subsubsection{Screen Readers}

Screen readers, for example JAWS ${ }^{\circledR}$, are software programs that verbalize everything on screen (e.g. graphics, text, punctuation, names and descriptions of buttons and menus) as speech (Nanopac Inc., 2003). A program of this sort will transform a GUI to an audio interface (using an internal speech synthesizer and sound card), and lessens the effort required to scan through the output interface

\subsubsection{Software Development Guidelines}

In order to achieve a truly accessible information society, many countries, international organisations and developers are putting forward guidelines to ensure that products are accessible and compatible. Development software must also have the capability of programming built-in accessibility features. These features must be able to accommodate visual, auditory or motor disabilities. If guidelines are adhered to during the development process, costs of modifying completed products will not be an added liability (TIA, 1996).

The following is a list of requirements that should, at a minimum, be demanded by customers and be taken into consideration by designers, developers and vendors of software products for quadriplegics and other users with mobility disabilities (IBM Accessibility Center, 2003; Nordic Guidelines for Computer Accessibility, 1998; TIA, 1996):

- Keyboard navigation: Provide mnemonics (accelerators) on graphical user interface components and functions; set tab order to standard top-2-bottom left-2-right (T2B, L2R) navigation; allow user navigation between and within menus, using the keyboard; equip all compo- nents with accessibility labels so that screen readers can verbalise them; and set the focus on the primary object that the user must operate. Screen readers also require the focus to be set in order to know which component to verbalise.

- User interfaces must be customisable.

- Provide redundant information for all objects: sound, text and graphics.

- Avoid interference with preset accessibility features such as sticky keys by not using keyboard sequences reserved for such features.

- Allow a time delay when requesting input from the user, as people with mobility disabilities may require more time for movement.

- Provide for compatibility with assistive technology. Provide accurate documentation for all accessibility features in a number of accessible formats, for example, having the documentation available in both an HTML format (which is more compatible with screen readers) and PDF format, and test completed software with a wide range of assistive technologies.

\subsubsection{Hardware Development Guidelines}

The following is a list of requirements that should, at a minimum, be demanded by customers and be taken into consideration by designers, developers and vendors of hardware products for quadriplegics and other users with mobility disabilities (IBM Accessibility Center, 2003; Nordic Guidelines for Computer Accessibility. 1998; TIA, 1996):

- Users should not have to grasp, simultaneously twist and turn, use twisting motions or press several controls simultaneously.

- Button sizes for pushbutton controls must be between 1.25 and $2.5 \mathrm{~cm}$ for finger activation, and 1.5 and $7.5 \mathrm{~cm}$ for palm activation.

- Separation distances for pushbutton controls must be $1.25 \mathrm{~cm}$ for finger activation and $5.0 \mathrm{~cm}$ for palm activation.

- Force required to activate pushbutton controls must not exceed 270 to $540 \mathrm{~g}$ for finger activation, or 270 to $2160 \mathrm{~g}$ for palm activation. (Other controls have similar minimum/maximum force, size or separation requirements).

- Texture of controls must be matte and non-slip, with redundant clues e.g. colour-coded, raised, with grooves, serrations or scallops.

- Components must not be made of materials that could trigger allergic reactions.

- Switch guards must be provided for critical switches as well as time-delays before activation.

- Keyboard keys and mouse buttons must be stiff enough to support resting fingers without being activated. 
- Ejected media such as diskettes and CDs must protrude at least $1.25 \mathrm{~cm}$.

- Provide load and unload ability for media via keyboard keys.

- Ensure that standard hardware and software interface properly with specialised assistive technologies.

- Security systems must provide alternative means of authorisation other than biometrics.

- Test hardware widely on people with varying degrees of limitations.

- Procurement guidelines: Developers must be required to declare which accessibility guidelines were adopted as the basis for their products. 'Request for proposals' and 'request for quotations' must explicitly indicate the accessibility features that vendors must include in their product in order to be allowed to tender for contracts.

\section{CONCLUSION}

This paper reported on an investigation into how quadriplegics use computer hardware and software, as well as technology to support this group of users. Some of the problems that they face were highlighted. Our study emphasised the fact that in order to successfully develop usable hardware and software for disabled persons, such as quadriplegics, they must be directly involved in the developments and testing of such technology. It also emphasised that if technology is adapted to suit disabled users, everybody can benefit, even ordinary able-bodied users. Some of the technology examples described in this paper was specifically developed with the disabled person in mind, but have found uses in various other application areas. Our paper concluded by providing guidelines for the development of computer software and hardware for quadriplegics. It is the basic human right of disabled persons to be able to interact effectively and efficiency with computer equipment. If support for such interaction is not provided, it can be seen as a form of technological 'apartheid'. It is hoped that designers will take note of the needs of disabled people, and design and build accessible technology to help the disabled to maximize their interaction with computers. The concept of universal design should be emphasised. Technology must be adapted to the needs of the users, and not vice versa.

The work reported upon in this article is partly based on work sponsored by a grant from the National Research Foundation of South Africa under Grant Number GUN: 2054025.

\section{REFERENCES}

Amyotrophic Lateral Sclerosis Association (ALSA). Retrieved April 2003 from http://www/alsa.org.

Arthritis Association (AA). Retrieved April 2003 from http://www.arthritis.com.

Children's Hemiplegia and Stroke Association (CHSA). Retrieved April 2003 from http://www.hemikids.org.

Christopher Reeve Paralysis Foundation (CRPF). Retrieved March 2003 from http://www. christopherreeve.org/ Research.

Dallabrida, D., 2002. Avoid the Mouse Trap. The News Journal. Retrieved April 2003 from www.delawareonline.com/newsjournal/life/2002/1119 avoidthemousetr.html

Grassie, W., 2001. A Low-Tech Obstacle to a Higher-Tech Future: The Keyboard Problem. Retrieved April 2003 from http://www.users.voicenet.com/ grassie/Fldr. Articles/Simplified.html.

IBM Accessibility Center. Retrieved April 2003 from http://www-3.ibm.com/able/.

Kotzé, P., 2000. Peopleware: Changing the Mindset of Computer Science and Engineering, Inaugural Lecture, Dept of Computer Science, UNISA, 19 October 2000.

Loy, B.A. \& Batiste, L.C., 2001 Speech Recognition: Options to Consider. Retrieved 15 February 2001 from http://www.jan.wvu.edu.

Marais, H., 2001. National Council for the Physically Disabled, Johannesburg, interviewed May 2001.

Metts, R. L., 2000. Disability Issues, Trends and Recommendations for the World Bank. Retrieved April 2003 from http://wbln0018.worldbank.org/HDNet/hddocs. nsf/2d5135ecbf351de68852566a90069b8b6/33ed2bea 9901edd6852568a20069e1c1/\$FILE/Metts.pdf.

Microsoft Accessibility. Retrieved May 2003 from http://www.microsoft.com/enable.

Mosby's Medical Encyclopaedia. 1997. The Learning Company Inc.

Multiple Sclerosis International Foundation (MSIF). Retrieved May 2003 from http://www.msif.org/ en/ms_the_disease/index.html.

Muscular Dystrophy Association of Canada (MDAC). Retrieved May 2003 from http://www.mdac.ca/.

Nanopac Inc. - Technology for Independence. Retrieved May 2003 from http://www.nanopac.com.

National Institute of Neurological Disorders and Strokes (NINDS). Retrieved May 2003 from http://www.ninds.nih.gov.

Nordic Guidelines for Computer Accessibility. 1998. Retrieved 24 April 2003 from http://trace.wisc .edu/docs/nordic_guidelines.htm.

The Parkinson's Web. Retrieved May 2003 from http://pdweb.mgh.harvard.edu.

Polk, C., 2000. Office machines everyone can use, Government Executive, 32 (9), 85- 86. 
Repetitive Stress Injury Association (RSIA). Retrieved May 2003 from, http://rsi.websitehostingservices.co.uk/headerpage1.asp

The Quadriplegic Association of South Africa (QASA). Retrieved May 2003 from http://quad.stormnet.co.za.

Sandhu, J., Gill, J. \& Martinez-Normand, L., 2003. Guidelines to Standardisers of ICT product and services in the CEN ICT domain - $3^{\text {rd }}$ draft. Retrieved December 2003 from http://www.tiresias/guidelines/ ceniss/.

Shneiderman, B., 1993. Sparks of Innovation in HumanComputer Interaction, Human-Computer Interaction Laboratory.

Shneiderman B., 2002. Universal Usability, Communications of the ACM, 43(5), $84-91$.

Telecommunications Industry Association (TIA), 1996. Resource Guide for Accessible Design of Consumer Electronics. Retrieved April 2003 from http://www.tiaonline.org/access/guide.html.

United Nations (UN). Report on a Priority Theme. Retrieved April 2003 from www.un.org/socdev/ disacc00.htm.

United Nations (UN) Statistics Division. Disability Statistics. Retrieved April 2003 from http://unstats.un/ rog/unsd/disability/.

Vanderheiden, G.C., 1994. Application Software Design Guidelines: Increasing the Accessibility of Application Software to People with Disabilities and Older Users. Retrieved May 2003 from http://trace.wisc.edu/docs/ software_guidelines/software.htm. 